\title{
Machine Learning and Deep Learning in Biomedical Engineering
}

\author{
Ghassan Khater Gzawi, Mohamed Fowzi Ababeneh \\ Royal Medical Services - Amman, Jordan \\ DOI: 10.29322/IJSRP.12.01.2022.p12132 \\ http://dx.doi.org/10.29322/IJSRP.12.01.2022.p12132
}

\begin{abstract}
Machine learning as well as Deep learning applications had qualified huge growth in different medical fields such as medical image analysis as well as other related data due to the fact of the convenience of several data sets to train the learning algorithms in multimodal modes. However, machine learning and deep learning can distinguish patterns in healthcare data to enhance diagnosis and prognosis. The most utilized machine learning and deep learning techniques for healthcare applications are autoencoder, restricted Boltzmann machine, deep belief network, recurrent neural network, convolutional neural network, generative adversarial network, neural networks, and support vector machine. This paper aimed to illustrate the different applications associated with these learning algorithms that focus on healthcare field. It also illustrates the high-tech methods utilized to employ the learning algorithms.
\end{abstract}

Index Terms- Machine learning, deep learning, image processing , and healthcare

\section{INTRODUCTION}

$\mathrm{M}$ achine learning, coined by Arthur Samuel, is a part of computer science which grants computers to learn deprived of being unequivocally programmed (Park et al., 2018). Having proceeded from the analysis of outline identification and computational learning hypothesis in artificial intelligence, machine learning establishes set of rules which can gather from a large body of data and get forecasts on the data. However, deep learning permits computational patterns with a lot of processing layers to learn a variety of degrees of abstraction for data representations. Deep learning utilizes the backpropagation procedure to exhibit how a machine have to adjust its internal factors that are employed to compute the interpretation in every layer from the interpretation in the previous layer, illuminating comprehensive form in big data sets (Julie et al., 2021). These methods (i.e., machine learning and deep learning) have considerably enhanced the high-tech in various biomedical fields such as prediction of deterioration of patients in ICU and/or general wards before the time of onset, prediction of mortality, and detection of breast cancer.

\section{FIELDS OF LEARNING ALGORITHMS IN BIOMEDICAL ENGINEERING}

This section aims to highlight the scope of a variety of presentations of machine learning and deep learning in the biomedical engineering area, as well as a particular prominence on the most characteristic learning models. Amongst the distinct concentrations of machine learning and deep learning, computer vision is considered an advanced stem of computer science which can perform entirely when merged with the different learning technologies (Wood et al., 2021). In computer vision, different problems were solved including face recognition, object detection and tracking, automatic document analysis, and medical image processing. Newly, the prompt advancements in innovative computing and imaging systems in biomedical engineering fields have given rise to a recent research aspect, and the expanding size of biomedical data necessitates accurate learning-based data mining algorithms. Not long ago, investigators have moved their emphasis on the way to employing digital image processing methods to perform different processes in brain tumors from Computed Tomography (CT) as well as Magnetic Resonance Imaging (MRI).

A recent paper entitled "Semantic segmentation using deep learning for brain tumor MRI via fully convolution neural networks" by Kumar et al, states that early head lump perception along with analysis might make serious medical problems to clinic (Kumar et al., 2019). In this research paper, two significant architectures for brain tumor segmentation were developed and their precision on pinnacle BraTS confront 2017 dataset were evaluated, along with as well investigate the role of transport knowledge beginning the BraTS architecture to the Rembrandt dataset. A number of outcomes pre-scented at this time deliberately, the make utilization patch-wise methodology to glioma dissection are tremendously capable. Potential engagement is expected to focal point on installing a more multidimensional FCN (Fully convolutional neural networks) architecture and conducts dice drubbing to collect the brat dataset and transfer understanding. Commonly, the paper addresses that by means of extra occupation, it will enlarge the undertaking to utilize convolutional neural network $(\mathrm{CNN})$ to competently and incompetently subdivision brain tumors, and in this manner, transport heaps of the application for instance surgical homework and mental representation quicker keen on arrive at.

The second paper entitled "High-sensitivity detection of facial features on MRI brain scans with a convolutional network" by Bansal et al. introduces a structure for distinguishing whether an 
input MRI brain scan has been disfigured, with the supreme objective of modernizing it within the compliance conventions of MRI data archiving and allocation stands (Bansal et al., 2021). The study provides a binary (defaced/"nondefaced") classifier according to a pattern convolutional neural network (CNN) architecture. The proposed model was trained on 980 de-faced MRI scans from 36 distinct studies that are widely presented at OpenNeuro.org. For overwhelm inaccessibility of nondefaced illustrations, the authors strengthen the dataset via inpainting artificial faces into every training image. The study showed the appropriateness of such a data reinforcement in a cross-validation evaluation.

This study also launches machine-learning approaches that were established for retinal image analysis. The third paper entitled "Deep-Learning-Based Automatic Computer-Aided Diagnosis System for Diabetic Retinopathy" by Romany F. Mansour tries to solve the classification problem in diabetic retinopathy (Mansour, 2018). In particular, the article offers the utilization of AlexNet deep neural networks (DNNs), that works based on convolutional neural network (CNN), to facilitate an optimum diabetic retinopathy computer-aided diagnosis solution. The experimental outcomes with standard KAGGLE fundus datasets expose that the suggested AlexNet DNN-based system demonstrates a superior implementation with LDA feature selection, where it displays a classification accuracy of $97.93 \%$. the good performance states that the anticipated model facilitates primary disease detection and diagnosis decision in diabetic retinopathy.

A recent published paper entitled "Prediction of mortality from 12-lead electrocardiogram voltage data using a deep neural network" theorized that a deep neural network (DNN) maybe able to forecast an important future clinical event, one-year all-cause mortality, from ECG voltage - time traces (Raghunath et al., 2020). The paper states that a forecast of risk is vital to the practice of medicine. Within cardiovascular medicine, for instance, risk scoring systems are extensively utilized to support a variability of significant distinct patient care decisions, for example defining the concentration of medical therapy, serving to adopt whether invasive therapies are defensible, deciding on the utilization of unconventional heart failure therapies, and controlling an ischemic workup before elective surgery. The acquired results of this paper show that deep learning can combine considerable prognostic information to the interpretation of 12-lead resting ECGs, just as in instances which are explained as standard via Doctor of Medicine.

Beritelli et al. recommends a paper entitled "Automatic Heart Activity Diagnosis based on Gram Polynomials and Probabilistic Neural Networks" offer a technique for diagnosing cardiac illness utilizing machine-learning methods like neural networks in their article (Boonzaaier, 2017). Using Gram polynomials to extract features, probabilistic neural networks were used to distinguish normal and abnormal heart sounds (phonocardiogram) from over 3000 data sets. Sleep analysis, like automated sleep staging and diagnosis of sleep disorders, has frequently used machine-learning algorithms. Another paper by Wei et al. (Wei et al., 2018), entitled "The Research of Sleep Staging Based on Single-lead Electrocardiogram and Deep Neural Network" develops a deep neural network (DNN) model applied utilizing a stacked autoencoder. The DNN structure classifies three sleep stages, wake, REM, and Non-REM, utilizing just one channel electrocardiogram (ECG) signal.

Dey et al. (Dey et al., 2018) proposes a paper entitled "Obstructive Sleep Apnoea Detection using convolutional neural network based deep learning framework" present an end-to-end convolutional neural network $(\mathrm{CNN})$ framework to identify obstructive sleep apnoea (OSA) using a single-lead ECG signal in their publication. While Wei et al. used traditional methods to extract features from raw ECG before applying the DNN classifier, Dey et al. fed the raw ECG signal straight into CNN and let the algorithm define the feature and class outputs concurrently.

\section{CONCLUSION}

Ultimately, systems that combine representation learning with sophisticated reasoning will make significant advances in artificial intelligence. Despite the fact that deep learning and basic reasoning have long been employed for different fields in biomedical engineering such as voice and handwriting identification, new paradigms are required to replace rule-based symbolic expression manipulation with operations on huge vectors.

\section{REFERENCES}

[1] Bansal, S., Kori, A., Zulfikar, W., Wexler, J., Markiewicz, C., Feingold, F., et al. (2021). High-sensitivity detection of facial features on MRI brain scans with a convolutional network. bioRxiv.

[2] Boonzaaier, D. (2017). Training a Neural Network for Checkers. Ph. D. thesis.

[3] Dey, D., Chaudhuri, S., and Munshi, S. (2018). Obstructive sleep apnoea detection using convolutional neural network based deep learning framework. Biomedical engineering letters, 8(1), 95-100.

[4] Julie, E. G., Robinson, Y. H., and Jaisakthi, S. (2021). Handbook of Deep Learning in Biomedical Engineering and Health Informatics: CRC Press.

[5] Kumar, S., Negi, A., and Singh, J. (2019). Semantic segmentation using deep learning for brain tumor MRI via fully convolution neural networks. In Information and Communication Technology for Intelligent Systems (pp. 1119): Springer.

[6] Mansour, R. F. (2018). Deep-learning-based automatic computer-aided diagnosis system for diabetic retinopathy. Biomedical engineering letters, 8(1), 41-57.

[7] Park, C., Took, C. C., and Seong, J.-K. (2018). Machine learning in biomedical engineering: Springer.

[8] Raghunath, S., Cerna, A. E. U., Jing, L., Stough, J., Hartzel, D. N., Leader, J. B., et al. (2020). Prediction of mortality from 12-lead electrocardiogram voltage data using a deep neural network. Nature medicine, 26(6), 886-891.

[9] Wei, R., Zhang, X., Wang, J., and Dang, X. (2018). The research of sleep staging based on single-lead electrocardiogram and deep neural network. Biomedical engineering letters, 8(1), 87-93.

[10] Wood, D. A., Kafiabadi, S., Al Busaidi, A., Guilhem, E. L., Lynch, J., Townend, M. K., et al. (2021). Deep learning to automate the labelling of head MRI datasets for computer vision applications. European Radiology, 112.

\section{AUTHORS}

First Author - Ghassan Khater Gzawi, Royal Medical Services - Amman, Jordan

Second Author - Mohamed Fowzi Ababeneh, Royal Medical Services - Amman, Jordan 
